\title{
Front Matter: Volume 7971
}

, "Front Matter: Volume 7971," Proc. SPIE 7971, Metrology, Inspection, and Process Control for Microlithography XXV, 797101 (6 May 2011); doi: $10.1117 / 12.898587$

SPIE. Event: SPIE Advanced Lithography, 2011, San Jose, California, United States 


\section{PROCEEDINGS OF SPIE}

\section{Metrology, Inspection, and Process Control for Microlithography XXV}

Christopher J. Raymond

Editor

28 February-3 March 2011

San Jose, United States

Sponsored and Published by

SPIE

Cosponsored by

Nova Measuring Instruments Ltd. (Israel)

Part One of Two Parts

Volume 7971 
The papers included in this volume were part of the technical conference cited on the cover and title page. Papers were selected and subject to review by the editors and conference program committee. Some conference presentations may not be available for publication. The papers published in these proceedings reflect the work and thoughts of the authors and are published herein as submitted. The publisher is not responsible for the validity of the information or for any outcomes resulting from reliance thereon.

Please use the following format to cite material from this book:

Author(s), "Title of Paper," in Metrology, Inspection, and Process Control for Microlithography XXV, edited by Christopher J. Raymond, Proceedings of SPIE Vol. 7971 (SPIE, Bellingham, WA, 2011) Article CID Number.

ISSN 0277-786X

ISBN 9780819485304

Published by

SPIE

P.O. Box 10, Bellingham, Washington 98227-0010 USA

Telephone +1 3606763290 (Pacific Time) · Fax +1 3606471445

SPIE.org

Copyright (C) 2011, Society of Photo-Optical Instrumentation Engineers

Copying of material in this book for internal or personal use, or for the internal or personal use of specific clients, beyond the fair use provisions granted by the U.S. Copyright Law is authorized by SPIE subject to payment of copying fees. The Transactional Reporting Service base fee for this volume is $\$ 18.00$ per article (or portion thereof), which should be paid directly to the Copyright Clearance Center (CCC), 222 Rosewood Drive, Danvers, MA 01923. Payment may also be made electronically through CCC Online at copyright.com. Other copying for republication, resale, advertising or promotion, or any form of systematic or multiple reproduction of any material in this book is prohibited except with permission in writing from the publisher. The CCC fee code is 0277-786X/11/ \$18.00.

Printed in the United States of America.

Publication of record for individual papers is online in the SPIE Digital Library.

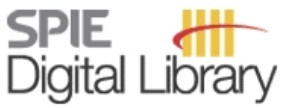

SPIEDigitalLibrary.org

Paper Numbering: Proceedings of SPIE follow an e-First publication model, with papers published first online and then in print and on CD-ROM. Papers are published as they are submitted and meet publication criteria. A unique, consistent, permanent citation identifier (CID) number is assigned to each article at the time of the first publication. Utilization of CIDs allows articles to be fully citable as soon as they are published online, and connects the same identifier to all online, print, and electronic versions of the publication. SPIE uses a six-digit CID article numbering system in which:

- The first four digits correspond to the SPIE volume number.

- The last two digits indicate publication order within the volume using a Base 36 numbering system employing both numerals and letters. These two-number sets start with 00, 01, 02, 03, 04, $05,06,07,08,09,0 A, 0 B \ldots 0 Z$, followed by 10-1Z, 20-2Z, etc.

The CID number appears on each page of the manuscript. The complete citation is used on the first page, and an abbreviated version on subsequent pages. Numbers in the index correspond to the last two digits of the six-digit CID number. 


\title{
Contents
}

\author{
xiii Conference Committee \\ xvii Introduction \\ xix The Diana Nyyssonen Memorial Award for Best Paper
}

\section{Part 1}

\section{SESSION 1 MASK AND LITHOGRAPHY METROLOGY}

797103 A holistic metrology approach: hybrid metrology utilizing scatterometry, CD-AFM, and CD-SEM (Invited Paper) [7971-02]

A. Vaid, B. B. Yan, Y. T. Jiang, M. Kelling, GLOBALFOUNDRIES Inc. (United States); C. Hartig, J. Allgair, P. Ebersbach, GLOBALFOUNDRIES Inc. (Germany); M. Sendelbach, N. Rana, A. Katnani, E. Mclellan, C. Archie, IBM Corp. (United States); C. Bozdog, H. Kim, M. Sendler,

S. Ng, Nova Measuring Instruments Inc. (United States); B. Sherman, B. Brill, I. Turovets, R. Urensky, Nova Measuring Instruments Ltd. (Israel)

797105 Litho process control via optimum metrology sampling while providing cycle time reduction and faster metrology-to-litho turn around time [7971-04]

K.-H. Chen, J. Huang, W.-T. Yang, C.-M. Ke, Y.-C. Ku, J. Lin, Taiwan Semiconductor Manufacturing Co. Ltd. (Taiwan); K. Bhattacharyya, E. Mos, M. Shahrjerdy, M. van der Schaar, S. Meyer, S. Lin, J. Wu, S. Peng, A. Li, N. Chang, R. Chu, C. Wang, ASML Netherlands B.V. (Netherlands)

797106 Mask registration impact on intrafield on-wafer overlay performance [7971-05] G.-T. Huang, A. Chen, T.-Y. Kang, S. C. Lee, Taiwan Semiconductor Manufacturing Co. Ltd. (Taiwan); F. Laske, K.-D. Roethe, KLA-Tencor Corp. (Germany); D. Choi, KLA-Tencor Corp. (Korea, Republic of); C. Reinhart, KLA-Tencor Corp. (Taiwan); J. C. Robinson, Y. S. Jin, L. Chua, D. Tien, V. R. Nagaswami, KLA-Tencor Corp. (United States)

797107 Application of mask process correction (MPC) to monitor and correct mask process drift [7971-06]

T. Lin, T. Donnelly, G. Russell, Mentor Graphics Corp. (United States); S. Jung, J. Jeong, Mentor Korea Co., Ltd. (Korea, Republic of)

\section{SESSION 2 SCANNING PROBE METROLOGY}

797108 Sub-nanometer line width and line profile measurement for CD-SEM calibration by using STEM (Invited Paper) [7971-07]

K. Takamasu, H. Okitou, S. Takahashi, The Univ. of Tokyo (Japan); M. Konno, O. Inove,

H. Kawada, Hitachi High-Technologies Corp. (Japan) 
797109 Challenges of SEM-based critical dimension metrology of interconnect [7971-08]

V. A. Ukraintsev, Nanometrology International, Inc. (United States); S. Jessen, B. Mikeska, Texas Instruments Inc. (United States); C. Sallee, V. Khvatkov, Smart Imaging Technologies (United States)

7971 OA Robust edge detection with considering three-dimensional sidewall feature by CD-SEM [7971-09]

A. Yamaguchi, H. Koyanagi, J. Tanaka, Hitachi, Ltd. (Japan); O. Inove, H. Kawada, Hitachi High-Technologies Corp. (Japan)

$7971 \mathrm{OB} \quad$ Tool-to-tool matching issues due to photoresist shrinkage effects [7971-10]

B. Bunday, A. Cordes, SEMATECH (United States); C. Hartig, J. Allgair, GLOBALFOUNDRIES Inc. (Germany); A. Vaid, GLOBALFOUNDRIES Inc. (United States); E. Solecky, N. Rana, IBM Microelectronics (United States)

7971 OC Influence of the charging effect on the precision of measuring EUV mask features [7971-11] Y. Nishiyama, H. Hakii, I. Yonekura, K. Tanaka, Y. Kikuchi, Toppan Printing Co., Ltd. (Japan)

\section{SESSION 3 INSPECTION}

7971 OD Optical illumination optimization for patterned defect inspection [7971-12]

B. M. Barnes, R. Quinthanilha, Y.-J. Sohn, H. Zhou, R. M. Silver, National Institute of Standards and Technology (United States)

7971 OE Wafer noise models for defect inspection [7971-13]

T. F. Crimmins, Intel Corp. (United States)

7971 OF Quantitative measurement of voltage contrast in SEM images for in-line resistance inspection of incomplete contact [7971-14] M. Matsui, T. Yano, T. Odaka, Hitachi, Ltd. (Japan)

7971 OG Characterization of EUV resists for defectivity at 32nm [7971-15] O. Montal, I. Dolev, M. Rosenzweig, K. Dotan, D. Meshulach, O. Adan, S. Levi, Applied Materials (Israel); M.-P. Cai, C. Bencher, C. S. Ngai, Applied Materials, Inc. (United States); C. Jehoul, D. Van Den Heuvel, E. Hendrickx, IMEC (Belgium)

$7971 \mathrm{OH} \quad$ OPC verification and hotspot management for yield enhancement through layout analysis [7971-16]

G. Yoo, J. Kim, T. Lee, A. Jung, H. Yang, D. Yim, S. Park, Hynix Semiconductor Inc. (Korea, Republic of); K. Maruyama, M. Yamamoto, NanoGeometry Research Inc. (Japan); A. Vikram, Anchor Semiconductor, Inc. (United States); S. Park, Daouxilicon Inc. (Korea, Republic of)

7971 Ol A new methodology for TSV array inspection [7971-17]

Y. Fujimori, T. Tsuto, Y. Kudo, T. Inove, Nikon Corp. (Japan); K. Okamoto, Nikon Corp. (Japan) and Osaka Univ. (Japan) 
$79710 \mathrm{~J} \quad$ Statistical-noise effect on power spectrum of line-edge and line-width roughness with long-range correlation [7971-18]

A. Hiraiwa, A. Nishida, MIRAI-Selete (Japan)

7971 OK Reduction of SEM noise and extended application to prediction of CD uniformity and its experimental validation [7971-19]

H. Kim, C. Hwang, S. Oh, J. Yeo, Y. Kim, SAMSUNG Electronics Co., Ltd. (Korea, Republic of)

7971 OM High-precision edge-roughness measurement of transistor gates using three-dimensional electron microscopy combined with marker-assisted image alignment [7971-21]

S. Ono, Semiconductor Leading Edge Technologies, Inc. (Japan); M. Yamane, Hitachi, Ltd. (Japan); M. Ogasawara, Hitachi High-Technologies Corp. (Japan); A. Katakami, J. Yugami, Semiconductor Leading Edge Technologies, Inc. (Japan); M. Koguchi, H. Shinada, Hitachi, Ltd. (Japan); H. Kakibayashi, Hitachi High-Technologies Corp. (Japan); K. Ikeda, Y. Ohji, Semiconductor Leading Edge Technologies, Inc. (Japan)

7971 ON Mueller matrix ellipsometry of artificial non-periodic line edge roughness in presence of finite numerical aperture [7971-22]

M. Foldyna, LPICM, Ecole Polytechnique, CNRS (France); T. A. Germer, National Institute of Standards and Technology (United States); B. C. Bergner, Spectrum Scientific, Inc. (United States)

\section{SESSION 5 DESIGN-BASED METROLOGY}

797100 A CD-gap-free contour extraction technique for OPC model calibration [7971-23] T. Shibahara, T. Minakawa, M. Oikawa, Hitachi, Ltd. (Japan); H. Shindo, H. Sugahara, Y. Hojyo, Hitachi High-Technologies Corp. (Japan)

7971 OP Fast and accurate calibration for OPC process-window model using inverse weight algorithm [7971-24]

A. Parikh, Texas Instruments Inc. (United States)

$79710 Q \quad$ Contact edge roughness (CER) characterization and modeling: effects of dose on CER and critical dimension (CD) variation [7971-25]

V.-K. Murugesan Kuppuswamy, V. Constantoudis, E. Gogolides, National Ctr. for Scientific Research Demokritos (Greece); A. Vaglio Pret, R. Gronheid, IMEC (Belgium)

\section{SESSION $6 \quad$ NEW DIRECTIONS}

7971 OS Hybrid CD metrology concept compatible with high-volume manufacturing [7971-27] J. Foucher, P. Faurie, L. Dourthe, CEA-LETI (France); B. Irmer, C. Penzkofer, Nanotools GmbH (Germany)

7971 OT TSOM method for semiconductor metrology [7971-28]

R. Attota, R. G. Dixson, J. A. Kramar, J. E. Potzick, A. E. Vladár, National Institute of Standards and Technology (United States); B. Bunday, SEMATECH (United States); E. Novak, Bruker Nano Surfaces Division (United States); A. Rudack, SEMATECH (United States) 
7971 OW Experimental validation of 2D profile photoresist shrinkage model (Invited Paper) [7971-30] B. Bunday, A. Cordes, SEMATECH (United States); A. Self, L. Ferry, A. Danilevsky, Hitachi High Technologies America, Inc. (United States)

7971 OX Surface modification of EUVL mask blanks by e-beam [7971-33]

A. J. Kadaksham, SEMATECH North (United States); T. Laursen, ASML US, Inc. (United States); T. Owen, J. Underwood, A. Rastegar, SEMATECH North (United States)

7971 OY High-throughput critical dimensions uniformity (CDU) measurement of two-dimensional (2D) structures using scanning electron microscope (SEM) systems [7971-31]

J. Fullam, C. Boye, T. Standaert, J. Gaudiello, IBM Corp. (United States); D. Tomlinson, TM2C (United States); H. Xiao, W. Fang, X. Zhang, F. Wang, L. Ma, Y. Zhao, J. Jau,

Hermes-Microvision, Inc. (United States)

$79710 Z$ Verification and extension of the MBL technique for photo resist pattern shape measurement [7971-32]

M. Isawa, Hitachi High-Technologies Corp. (Japan); M. Tanaka, Hitachi, Ltd. (Japan); H. Kazumi, Hitachi High-Technologies Corp. (Japan); C. Shishido, A. Hamamatsu, Hitachi, Ltd. (Japan); N. Hasegawa, Hitachi High-Technologies Corp. (Japan); P. De Bisschop,

D. Laidler, P. Leray, S. Cheng, IMEC (Belgium)

797110 A method for improving resolution of a scanning electron microscope for inspection of nanodevices [7971-34]

M. Fukuda, N. Takahashi, T. Shojo, H. Ohta, H. Suzuki, Hitachi, Ltd. (Japan)

\section{SESSION 8 SCATTEROMETRY}

$797111 \quad$ Metrology characterization of spacer double patterning by scatterometry [7971-35] P. Dasari, J. Li, J. Hu, Z. Liu, Nanometrics Inc. (United States); O. Kritsun, C. Volkman, GLOBALFOUNDRIES Inc. (United States)

797112 Optical far field measurements applied to microroughness determination of periodic microelectronic structures [7971-36]

A. Vauselle, Institut Fresnel, CNRS, Aix-Marseille Univ. (France) and STMicroelectronics (France); P. Maillot, STMicroelectronics (France); G. Georges, C. Deumié, Institut Fresnel, CNRS, Aix-Marseille Univ. (France)

797113 A holistic metrology approach: multi-channel scatterometry for complex applications [7971-37]

C. Bozdog, H. K. Kim, S. Emans, Nova Measuring Instruments Inc. (United States);

B. Sherman, I. Turovets, R. Urensky, B. Brill, Nova Measuring Instruments Ltd. (Israel); A. Vaid, GLOBALFOUNDRIES (United States); M. Sendelbach, IBM Corp. (United States)

797114 Diffraction based overlay re-assessed [7971-38]

P. Leray, D. Laidler, K. D'havé, S. Cheng, IMEC (Belgium)

797115 Overlay measurements by Mueller polarimetry in the back focal plane [7971-39] T. Novikova, C. Fallet, M. Foldyna, S. Manhas, B. Haj Ibrahim, A. De Martino, LPICM, Ecole Polytechnique, CNRS (France); C. Vannuffel, C. Constancias, CEA-LETI (France) 
$797116 \quad$ Nested uncertainties and hybrid metrology to improve measurement accuracy [7971-40] R. M. Silver, N. F. Zhang, B. M. Barnes, H. Zhou, J. Qin, R. Dixson, National Institute of Standards and Technology (United States)

797117 Reconciling measurements in AFM reference metrology when using different probing techniques [7971-41]

N. Rana, C. Archie, IBM Corp. (United States); J. Foucher, CEA-LETI (France)

797118 New three-dimensional AFM for CD measurement and sidewall characterization [7971-42]

Y. Hua, C. Buenviaje-Coggins, Park Systems Inc. (United States); Y. Lee, J. Lee, K. Ryang,

S. Park, Park Systems Corp. (Korea, Republic of)

797119 High-speed atmospheric imaging of semiconductor wafers using rapid probe microscopy [7971-43]

P. Kohli, J. Lyons, A. D. L. Humphris, Infinitesima Ltd. (United Kingdom); B. D. Bunday, A. Arceo, A. Hamaguchi, D. Patel, SEMATECH North (United States); D. Bakker, Consultant (United Kingdom)

7971 1A Artifacts of the AFM image due to the probe controlling parameters [7971-44] $\mathrm{H}$. Itoh, C. Wang, H. Takagi, National Institute of Advanced Industrial Science and Technology (Japan)

\section{SESSION 10 INNOVATIVE LITHOGRAPHY PROCESS CONTROL: JOINT SESSION WITH CONFERENCE 7973}

7971 1C High sensitive and fast scanner focus monitoring method using forbidden pitch pattern [7971-46]

J. Heo, J.-H. Yeo, Y. Kim, SAMSUNG Electronics Co., Ltd. (Korea, Republic of)

\section{Part 2}

\section{SESSION 11 OVERLAY}

7971 1D Overlay improvement roadmap: strategies for scanner control and product disposition for 5-nm overlay [7971-47]

N. M. Felix, A. H. Gabor, V. C. Menon, P. P. Longo, S. D. Halle, C. Koay, M. E. Colburn, IBM Corp. (United States)

7971 1E Accuracy of diffraction-based and image-based overlay [7971-49] C.-M. Ke, G.-T. Huang, J. Huang, R. Lee, Taiwan Semiconductor Manufacturing Co. Ltd. (Taiwan)

7971 if Investigation on accuracy of process overlay measurement [7971-50]

C. Hwang, J. Lee, S. Lee, J. Yeo, Y. Kim, SAMSUNG Electronics Co., Ltd. (Korea, Republic of); H. Lim, KLA-Tencor Singapore (Singapore); D. Choi, KLA-Tencor Korea (Korea, Republic of) 
7971 IG Improved overlay control using robust outlier removal methods [7971-51] J. C. Robinson, KLA-Tencor Corp. (United States); O. Fujita, H. Kurita, KLA-Tencor Corp. Japan (Japan); P. Izikson, D. Klein, I. Tarshish-Shapir, KLA-Tencor Corp. Isreal (Israel)

7971 1H Wafer quality analysis of various scribe line mark designs [7971-52]

J. Zhou, C. Hickman, Y. He, S. Light, L. Lamonds, A. deVilliers, Micron Technology, Inc. (United States)

\section{SESSION 12 LITHOGRAPHY PROCESS CONTROL}

7971 1M Overlay and focus stability control for 28-nm nodes on immersion scanners [7971-57] G.-T. Huang, K.-H. Chen, L.-J. Chen, T.-S. Gau, Taiwan Semiconductor Manufacturing Co. Ltd. (Taiwan); R. Jungblut, A. Chen, E. Lee, L. Wang, M. Un, W.-S. Tzeng, J. Chen, S. Lin, J. Wu, ASML Taiwan Ltd (Taiwan)

$7971 \mathrm{lN}$ Towards $22 \mathrm{~nm}$ : fast and effective intra-field monitoring and optimization of process windows and CDU [7971-58]

Y. Cohen, Applied Materials (Israel); J. Finders, R. Knops, O. Mouraille, I. Minnaert-Janssen, F. Duray, E. Mos, A. Kremer, ASML Netherlands B.V. (Netherlands); A. Sagiv, S. Mangan, M. Ben Yishay, H. Dai, C. Bencher, C. Ngai, K. Dotan, I. Englard, Applied Materials (Israel)

797110 Automatic optimization of metrology sampling scheme for advanced process control [7971-60]

C.-F. Chue, C.-Y. Huang, C.-L. Shih, Nanya Technology Corp. (Taiwan)

7971 IP CD uniformity improvement of through-pitch contact-hole patterning for advanced logic devices [7971-62]

T. Kuribayashi, Y. Matsui, K. Yoshimochi, S. Nagahara, T. Uchiyama, Renesas Electronics Corp. (Japan)

\section{POSTER SESSION}

7971 IQ Sensitivity of LWR and CD linearity to process conditions in active area [7971-20] G. Ayal, Applied Materials, Inc. (Israel) and Tel Aviv Univ. (Israel); E. Malkes, E. Aharoni, Tower Semiconductor Ltd. (Israel); S. Levi, A. Siany, Applied Materials (Israel); O. Adan, Applied Materials (Israel) and Tel Aviv Univ. (Israel); E. Shauly, Tower Semiconductor Ltd. (Israel) and Technion-Israel Institute of Technology (Israel); Y. Shacham-Diamand, Tel Aviv Univ. (Israel)

7971 IR Real-time defection system of defects on a photo mask by using the light scattering and interference method [7971-63]

J. H. Jo, S. Lee, Hannam Univ. (Korea, Republic of); H. S. Wee, J. S. Kim, Nano Electro Optics Co., Ltd. (Korea, Republic of)

7971 is Improved secondary electron extraction efficiency model for accurate measurement of narrow-space patterns using model-based library matching [7971-64]

C. Shishido, M. Tanaka, A. Hamamatsu, Hitachi, Ltd. (Japan)

7971 iT Scatterometry simulator using GPU and evolutionary algorithm [7971-65]

H. Shirasaki, Tamagawa Univ. (Japan) 
7971 iU Simulation of non-uniform wafer geometry and thin film residual stress on overlay errors [7971-66]

S. Veeraraghaven, KLA-Tencor Corp. (United States); K. T. Turner, Univ. of Wisconsin-Madison (United States); J. Sinha, KLA-Tencor Corp. (United States)

$79711 \mathrm{~V} \quad$ Study of scanner stage vibration by using scatterometry [7971-67]

D. Jiang, W. Zhou, M. Hsieh, Q. Lin, GLOBALFOUNDRIES (Singapore)

7971 1Y Advancements of diffraction-based overlay metrology for double patterning [7971-70] J. Li, Nanometrics Inc. (United States); O. Kritsun, GLOBALFOUNDRIES Inc. (United States); Y. Liu, P. Dasari, U. Weher, Nanometrics Inc. (United States); C. Volkman, GLOBALFOUNDRIES Inc. (United States); M. Mazur, GLOBALFOUNDRIES (Germany); J. Hu, Nanometrics Inc. (United States)

797112 CD-SEM image-distortion measured by view-shift method [7971-71] O. Inove, T. Kawasaki, Hitachi High-Technologies Corp. (Japan); M. Matsui, Hitachi, Ltd. (Japan); H. Kawada, Hitachi High-Technologies Corp. (Japan)

797120 Scatterometry for EUV lithography at the 22-nm node [7971-72] B. Bunday, V. Vartanian, L. Ren, G. Huang, C. Montgomery, W. Montgomery, SEMATECH (United States); A. Elia, X. Liu, Timbre Technologies, Inc. (United States)

797121 Study of the three-dimensional shape measurement for mask patterns using Multiple Detector CD-SEM [7971-73]

I. Yonekura, H. Hakii, M. Kawashita, Y. Nishiyama, K. Tanaka, Y. Kikuchi, Toppan Printing Co., Ltd. (Japan); T. Murakawa, S. Shida, M. Kuribara, T. Iwai, J. Matsumoto, T. Nakamura, Advantest Corp. (Japan)

797123 EB defect inspection of EUV resist patterned wafer for hp $32 \mathbf{~ n m}$ and beyond [7971-76] M. Nozoe, T. Tanaka, T. Kamo, S. Kubo, T. Tamori, N. Takagi, T. Yamane, T. Terasawa, H. Shigemura, O. Suga, Semiconductor Leading Edge Technologies, Inc. (Japan)

797124 The study of high-sensitivity metrology method by using CD-SEM [7971-77] K. Ueda, S. Koshihara, T. Mizuno, A. Miura, Hitachi High-Technologies Corp. (Japan)

797126 EUV defect characterization study on post litho and etch for $1 x$ and $2 x$ node processes [7971-79]

O. Montal, Applied Materials (Israel); M.-P. Cai, K. Dotan, Applied Materials (United States); I. Dolev, Applied Materials (Israel); T. Wallow, O. Wood, U. Okoroanyanwu, GLOBALFOUNDRIES Inc. (United States); M. Rozentsvige, Applied Materials (Israel); C. Ngai, C. Bencher, Applied Materials, Inc. (United States); A. Conley, Applied Materials (Israel)

$797127 \quad$ High-order stitching overlay analysis for advanced process control [7971-80] Y. C. Pai, C. Chen, L. Jang, H. Chen, C.-C. Yu, United Microelectronics Corp. (Taiwan); C.-C. K. Huang, H.-C. Wu, J. C. Robinson, D. Tien, KLA-Tencor Corp. (United States)

797128 Influence of BARC filtration and materials on the reduction of spire defects [7971-82] J. Schneider, S. Volkland, U. Feldner, L. O'Riain, D. Peters, F. Braun, L. Brencher, B. Hornig, O. Luxenhofer, D. Sarlette, Infineon Technologies Dresden (Germany) 
797129 Novel CD-SEM magnification calibration reference of sub-50-nm pitch multi-layer grating with positional identification mark [7971-83]

Y. Nakayama, J. Yamamoto, Hitachi, Ltd. (Japan); O. Inove, H. Kawada, S. Yoneda, Hitachi High-Technologies Corp. (Japan)

7971 2A Expanding the applications of computational lithography and inspection (CLI) in mask inspection, metrology, review, and repair [7971-85]

L. Pang, D. Peng, P. Hu, D. Chen, L. He, Y. Li, C. Clifford, V. Tolani, Luminescent Technologies, Inc. (United States)

$79712 B \quad$ Methodology for overlay mark selection [7971-86]

C.-C. Huang, C.-T. Huang, A. Golotsvan, D. Tien, KLA-Tencor Corp. (United States);

C.-F. Chiu, C.-Y. Huang, W.-B. Wu, C.-L. Shih, Nanya Technology Corp. (Taiwan)

7971 2C Process solutions for reducing PR residue over non-planar wafer [7971-87]

C. H. Lin, C. H. Huang, E. Yang, T. H. Yang, K. C. Chen, C.-Y. Lu, Macronix International Co., Ltd. (Taiwan)

$79712 \mathrm{D}$ Impact of pellicle on overlay in double patterning lithography [7971-88]

O. Loeffler, Advanced Mask Technology Ctr. GmbH Co. KG (Germany); F. Laske, M. Ferber, K.-D. Roeth, KLA-Tencor MIE GmbH (Germany); L. Chua, Y. S. Jin, G. Marcuccilli,

V. Nagaswami, KLA-Tencor Corp. (United States)

$79712 \mathrm{E}$ 3D features measurement using YieldStar, an angle resolved polarized scatterometer [7971-89]

A.-L. Charley, P. Leray, K. D'havé, S. Cheng, IMEC (Belgium); P. Hinnen, F. Li, P. Vanoppen,

M. Dusa, ASML Netherlands B.V. (Netherlands)

$79712 \mathrm{G}$ The assessment of the impact of mask pattern shape variation on the OPC-modeling by using SEM-Contours from wafer and mask [7971-92]

D. Hibino, Y. Hojyo, H. Shindo, Hitachi High-Technologies Corp. (Japan); T. Do, A. Dave,

T. Lin, I. Kusnadi, J. L. Sturtevant, Mentor Graphics Corp. (United States)

$79712 \mathrm{H} \quad$ Calibration studies of pattern top resist loss detection by CD-SEM for advanced lithography process [7971-93]

T. Ishimoto, Hitachi High Technologies Corp. (Belgium); M. Isawa, Hitachi High-Technologies

Corp. (Japan); M. Tanaka, Hitachi, Ltd. (Japan); S. Cheng, IMEC (Belgium)

$797121 \quad$ Approaches to airborne molecular contamination assessment [7971-94]

S. Riddle Vogt, SAES Pure Gas, Inc. (United States); C. Landoni, SAES Getters S.p.A. (Italy)

$79712 \mathrm{~J}$ Metrology of micro-step height structures using 3D scatterometry in 4X-nm advance DRAM [7971-95]

M. Duan, Inotera Memories Inc. (Taiwan); C. Chen, C. Hsu, KLA-Tencor Corp. (United States); E. Wang, Z. XU, Inotera Memories Inc. (Taiwan); E. YU, Q. Yuan, S. Yoo, Z. Tan, KLA-Tencor Corp. (United States)

$79712 \mathrm{~K}$ A study and simulation of the impact of high-order aberrations to overlay error distribution [7971-96]

G. Sun, F. Wang, C. Zhou, Shanghai Micro Electronics Equipment Co., Ltd. (China) 
7971 2L Wafer-edge defect reduction for tri-layer materials in BEOL applications [7971-97] J. R. Du, C. H. Huang, E. Yang, T. H. Yang, K. C. Chen, C.-Y. Lu, Macronix International Co., Ltd. (Taiwan)

$79712 \mathrm{M}$ Enhanced defect of interest [DOI] monitoring by utilizing sensitive inspection and ADRTrue SEM review [7971-98]

R. Kirsch, U. Zeiske, GLOBALFOUNDRIES (Germany); S. Shabtay, M. Beyer, L. Yerushalmi,

O. Goshen, Applied Materials Israel (Israel)

$79712 \mathrm{~N} \quad$ Multifeature focus exposure matrix for tool diagnosis [7971-99]

Z. Yang, A. Y. Burov, L. Duan, F. Wang, Shanghai Micro Electronics Equipment Co., Ltd. (China)

797120 Scatterometry measurement for gate ADI and AEl critical dimension of 28-nm metal gate technology [7971-100]

Y.-H. Huang, H. Chen, K. Shen, H. H. Chen, C. C. YU, J. H. Liao, United Microelectronics Corp. (Taiwan); X. Zhang, R. Teo, Z.-Q. J. Xu, S. Yoo, C.-H. B. Lin, C.-Y. H. Cheng, J. Lin, KLA-Tencor Corp. (United States)

7971 2P Surface scanning inspection system defect classification of CMP induced scratches [7971-102]

S. McGarvey, Hitachi High Technologies America, Inc. (United States); A. E. Miller, Fujimi Corp. (United States)

$79712 Q \quad$ RS-Mini: an enterprise class highly compact mask inspection defect management framework for the mask and wafer fab infrastructure [7971-103]

S. Munir, Reticle Labs. (United States)

7971 2R Wavefront measurement for EUV lithography system through Hartmann sensor [7971-104] A. Polo, F. Bociort, S. F. Pereira, H. P. Urbach, Technische Univ. Delft (Netherlands)

$79712 \mathrm{U}$ Diffraction-based overlay for spacer patterning and double patterning technology [7971-107]

B. H. Lee, J. Park, J. Lee, S. Park, C. Lim, D.-G. Yim, S. Park, Hynix Semiconductor Inc. (Korea, Republic of); C.-H. Ryu, S. Morgan, M. van de Schaar, A. Fuchs, K. Bhattacharyya, ASML

Netherlands B.V. (Netherlands)

$79712 \mathrm{~V}$ Automated CD-SEM recipe creation technology for mass production using CAD data [7971-109]

T. Kawahara, Hitachi High-Technologies Corp. (Japan); M. Yoshida, M. Tanaka, S. Ido, H. Nakano, Sony Semiconductor Kyushu Corp. (Japan); N. Adachi, Y. Abe, W. Nagatomo, Hitachi High-Technologies Corp. (Japan)

Author Index 
Downloaded From: https://www.spiedigitallibrary.org/conference-proceedings-of-spie on 25 Apr 2023

Terms of Use: https://www.spiedigitallibrary.org/terms-of-use 


\title{
Conference Committee
}

\author{
Symposium Chairs
}

Donis G. Flagello, Nikon Research Corporation of America (United States)

Harry J. Levinson, GLOBALFOUNDRIES Inc. (United States)

Conference Chair

Christopher J. Raymond, Nanometrics Inc. (United States)

Conference Cochair

Alexander Starikov, I\&I Consulting (United States)

Program Committee

Ofer Adan, Applied Materials (Israel)

John A. Allgair, GLOBALFOUNDRIES, Inc. (Germany)

Chas N. Archie, IBM Microelectronics Division (United States)

Masafumi Asano, Toshiba Corporation (Japan)

Benjamin D. Bunday, International SEMATECH Manufacturing Initiative (United States)

Jason P. Cain, Advanced Micro Devices, Inc. (United States)

Shaunee Y. Cheng, IMEC (Belgium)

Daniel J. C. Herr, Semiconductor Research Corporation (United States)

David C. Joy, The University of Tennessee (United States)

Chih-Ming Ke, Taiwan Semiconductor Manufacturing Company Ltd.

(Taiwan)

Yi-sha Ku, Industrial Technology Research Institute (Taiwan)

Byoung-Ho Lee, SAMSUNG Electronics Company, Ltd.

(Korea, Republic of)

John C. Robinson, KLA-Tencor Corporation (United States)

Martha I. Sanchez, IBM Almaden Research Center (United States)

Richard M. Silver, National Institute of Standards and Technology

(United States)

Vladimir A. Ukraintsev, Nanometrology International, Inc.

(United States) 
Keynote Session

Christopher J. Raymond, Nanometrics Inc. (United States)

Alexander Starikov, I\&I Consulting (United States)

Mask and Lithography Metrology

Alexander Starikov, I\&I Consulting (United States)

Masafumi Asano, Toshiba Corporation (Japan)

Scanning Probe Metrology

Martha I. Sanchez, IBM Almaden Research Center (United States)

Byoung-Ho Lee, SAMSUNG Electronics Company, Ltd.

(Korea, Republic of)

Inspection

Jason P. Cain, Advanced Micro Devices, Inc. (United States)

John C. Robinson, KLA-Tencor Corporation (United States)

LER/LWR

Martha I. Sanchez, IBM Almaden Research Center (United States)

Benjamin D. Bunday, International SEMATECH Manufacturing Initiative (United States)

Design-based Metrology

Masafumi Asano, Toshiba Corporation (Japan)

John A. Allgair, GLOBALFOUNDRIES, Inc. (Germany)

New Directions

Vladimir A. Ukraintsev, Nanometrology International, Inc.

(United States)

Daniel J. C. Herr, Semiconductor Research Corporation (United States)

SEM

Ofer Adan, Applied Materials (Israel)

Byoung-Ho Lee, SAMSUNG Electronics Company, Ltd.

(Korea, Republic of)

Scatterometry

Christopher J. Raymond, Nanometrics Inc. (United States)

Jason P. Cain, Advanced Micro Devices, Inc. (United States) 
AFM and Standards

Vladimir A. Ukraintsev, Nanometrology International, Inc.

(United States)

Benjamin D. Bunday, International SEMATECH Manufacturing Initiative (United States)

Innovative Lithography Process Control: Joint Session with

Conference 7973

Will Conley, Freescale Semiconductor, Inc. (United States)

Alexander Starikov, I\&I Consulting (United States)

Overlay

Richard M. Silver, National Institute of Standards and Technology (United States)

Shaunee Y. Cheng, IMEC (Belgium)

X-ray and Novel Methods

Richard M. Silver, National Institute of Standards and Technology (United States)

David C. Joy, The University of Tennessee (United States)

Lithography Process Control

Shaunee Y. Cheng, IMEC (Belgium)

Ofer Adan, Applied Materials (Israel)

Poster Session

Jason P. Cain, Advanced Micro Devices, Inc. (United States)

John C. Robinson, KLA-Tencor Corporation (United States) 
Downloaded From: https://www.spiedigitallibrary.org/conference-proceedings-of-spie on 25 Apr 2023

Terms of Use: https://www.spiedigitallibrary.org/terms-of-use 


\section{Introduction}

The conference on Metrology, Inspection and Process Control for Microlithography:

With contributions from more than ten thousand authors, comprising more than three thousand technical papers, spanning twenty five years and hosted at two venues, there has been one conference bringing you the latest advances in metrology for the silicon industry and beyond. It is my pleasure to introduce the proceedings for the $25^{\text {th }}$ anniversary of Metrology, Inspection and Process Control for Microlithography.

It is my distinct honor to be the chair of "Metrology 25" as it celebrates its silver anniversary. The conference had humble beginnings in small hotel rooms in the 1980s, where Diana Nyyssonen established earlier conferences on metrology development at the National Bureau of Standards (NBS), and unofficially dates "Metrology" beyond twenty five years of age. Since then the conference has become a staple of Advanced Lithography, with good attendance, strong technical contributions and high paper counts. Looking back it is clear that colocating lithography, metrology and resist conferences in Silicon Valley may be the root of the highly symbiotic relationships that benefitted everyone involved. In addition to acknowledging Diana's role as the metrology pioneer, educator and promoter, I would like to thank the early conference chairs for their vision and contributions, particularly Kevin Monahan and Michael Postek, who gave well received personal recollections that reflected on conference history during our opening session remarks this past year.

I also want to acknowledge and thank Bob McNeil, who led the University of New Mexico (UNM) scatterometry effort in the 1990s and was a regular at this conference during that era. Sadly Bob passed away earlier this year. Bob was my Ph.D. advisor at UNM, and in addition to being an excellent mentor, I also considered him to be a good friend. As an early pioneer in scatterometry he leaves a considerable legacy on this conference, but he also made strong contributions to the broader field of optics through his work in laser development, thin film coatings, optical roughness measurements and novel display technologies. Yet more important than anything, he was a true gentleman.

Finally, I wish to acknowledge the hard work of my program committee over the last two years. I also wish to extend special thanks to the staff at SPIE for all that they do to make the larger symposium a success year after year. 
I am looking forward to Metrology 50! In the meantime, I hope to see you, your colleagues and your paper at Metrology, Inspection and Process Control for Microlithography XXVI!

Christopher J. Raymond 


\section{The Diana Nyyssonen Memorial Award for Best Paper}

Diana Nyyssonen was a pioneer of dimensional metrology in microelectronics. Through her work at the National Bureau of Standards (NBS), she discovered the resolution of metrology with an optical microscope is much better than classical limits. Diana developed numerical models and became the first to make model-based measurements on photomasks, establishing the earliest critical dimension standards for our industry and the foundations of model-based metrology. Her work on optical edge detection and the imaging of thick layers, and advocacy for detailed modeling of the metrology process itself, invigorated dimensional metrology. She started the SPIE Conferences on Integrated Circuit Metrology in Arlington, Virginia in 1982.

Diana left NBS to form her own metrology company and later joined IBM at East Fishkill, New York. Her attention shifted from conventional microscopy to phase and amplitude imaging with interference microscopy. She also modeled secondary electron images for low voltage CD-SEM, and defined the requirements for AFM tips and sensing methods to enable CD-AFM.

Diana received her Ph.D. from the Institute of Optics, University of Rochester. Her personal contributions to the field and her many collaborations with metrology vendors, standards laboratories, consortia and academia, accelerated and broadly influenced the development of technology infrastructure and metrology applications.

As a part of SPIE's Advanced Lithography Symposium, the Metrology, Inspection and Process Control Conference is the leading international forum for the discussion and presentation of technical advances in the broader field of semiconductor metrology. The Diana Nyyssonen Memorial Award for the Best Paper at this conference was established to recognize the most significant current contribution to semiconductor manufacturing and metrology for process control.

Due to the conference's long history, significant attendance and high paper counts, to win this Award requires a very significant new contribution to the field. The selection of the best paper is initiated during the conference by nomination, followed by extensive review by the program committee. It is based on both the technical merit and persuasiveness of the oral presentation as well as the overall quality of the published paper. Recent winners include leading international researchers in the area of semiconductor metrology and process control whose contributions have fundamentally improved the way semiconductors are manufactured.

We are pleased to honor the winners of the Diana Nyyssonen Memorial Award for the Best Paper of 2010, as well as others who have won in previous years. 


\section{0}

David Laidler, Koen D'havé, Anne-Laure Charley, Shaunee Cheng, Philippe Leray, Peter Vanoppen, Mircea Dusa, Paul Hinnen, "A single metrology tool solution for complete exposure tool setup"; Proc. SPIE, Vol. 7638, 763809 (2010).

\section{9}

Benjamin Bunday, Aaron Cordes, John Allgair, Vasiliki Tileli, Yohanan Avitan, Ram Peltinov, Maayan Bar-zvi, Ofer Adan, Eric Cottrell, Sean Hand, "Phenomenology of electron-beam-induced photoresist shrinkage trends"; Proc. SPIE, Vol. 7272, 72721B (2009).

\section{8}

Alok Vaid and Rohit Pal, Matthew Sendelbach, Shahin Zangooie, Kevin Lensing, Carsten Hartig, "Scatterometry as technology enabler for embedded SiGe process"; Proc. SPIE, Vol. 6922, 69220 U (2008).

\section{7}

Matthew Sendelbach, Javier Ayala, Pedro Herrera, "Predicting electrical measurements by applying scatterometry to complex spacer structures"; Proc. SPIE, Vol. 6518, 651825 (2007).

\section{6}

Masafumi Asano, Takahiro Ikeda, Toru Koike, Hideaki Abe, "In-line CD metrology with combined use of scatterometry and CD-SEM"; Proc. SPIE, Vol. 6152, 61521V (2006).

\section{5}

John S. Villarrubia, Benjamin D. Bunday, "Unbiased estimation of linewidth roughness"; Proc. SPIE, Vol. 5752, 480 (2005).

\section{4}

Christopher P. Ausschnitt, "A new approach to pattern metrology"; Proc. SPIE, Vol. 5375,51 (2004).

\section{3}

John S. Villarrubia, Andras E. Vladar, Michael T. Postek, "Simulation study of repeatability and bias in the CD-SEM"; Proc. SPIE, Vol. 5038, 138 (2003).

\section{2}

Jon L. Opsal, Hanyou Chu, Youxian Wen, Yia-Chung Chang, Guangwei Li, "Fundamental solutions for real-time optical CD metrology"; Proc. SPIE, Vol. 4689, 163 (2002).

\section{1}

Sylvain Muckenhirn, A. Meyyappan, Kelvin Walch, Mark J. Maslow, Geert Vandenberghe, Johannes van Wingerden, "SPM characterization of anomalies in phase-shift mask and their effect on wafer features; Proc. SPIE, Vol. 4344, 188 (2001). 